Article

\title{
Purification of Amygdalin from the Concentrated Debitterizing-Water of Apricot Kernelsusing XDA-1 Resin
}

\author{
Qing-An Zhang *(D), Dong-Dong Wu and Chen-Xi Wei \\ Institute of Food \& Physical Field Processing, School of Food Engineering and Nutrition Sciences, \\ Shaanxi Normal University, Xi'an 710062, China; wudong@snnu.edu.cn (D.-D.W.); \\ chenby@snnu.edu.cn (C.-X.W.) \\ * Correspondence: qinganzhang@snnu.edu.cn; Tel./Fax: +86-29-85310517
}

Received: 16 May 2019; Accepted: 3 June 2019; Published: 10 June 2019

\begin{abstract}
In this study, six macroporous resins were screened on their adsorption and de-adsorption characteristics for the amygdalin in the debitterizing wastewater concentrate (DWC). The results indicate that the XDA-1 resin exerts good adsorption and de-adsorption capacities on the amygdalin. In order to further confirm its feasibility, the factors affecting the capacity of adsorption and de-adsorption, and its adsorption mechanisms were also investigated. The results suggest that the optimum purification conditions were as follows: loading concentration of samples with $78.05 \mathrm{mg} / \mathrm{mL}$, flow rate of $2 \mathrm{~mL} / \mathrm{min}$, and de-adsorption with $80 \%$ ethanol solution. The recovery rate was $88.75 \%$ and the relative content achieved $61.58 \%$ after purification by XDA- 1 resin. The Freundlich model can be used to describe the entirety of the exothermic and physical adsorption processes. In summary, the conclusion which can be made from this research is that the wastewater generated from the debitterizing of apricot kernels can be well treated by resin to recycle the amygdalin and reduce its pollution to environment.
\end{abstract}

Keywords: apricot kernels; debitterizing water; amygdalin; separation; macroporous resins; macroporous resins

\section{Introduction}

Apricot (Prunus armeniaca L.) is a globally popular fruit crop due to its delicious taste, appealing smell and color [1]. With the gradually increasing consumption of the apricot, many apricot kernels are generated as a significant by-product [2]. Apricot kernels contain many nutritional components, such as oil, proteins, fibers [3,4], carbohydrates, vitamins [5], unsaturated fatty acids, phytosterols [4], carotenoids, phenolic compounds [2], minerals [6], and so on.

Amygdalin is a characteristic component of the apricot kernels, which has an approximate content of $3-5 \%(\mathrm{~g} / 100 \mathrm{~g})$ [7]. As a cyanogenic diglucoside, the amygdalin can be hydrolyzed to hydrogen cyanide (HCN) in the body, which is a potentially toxigenic substance [8]. Thus, the removal of amygdalin (debitterizing) is a necessary procedure in apricot kernel processing [9]. The conventional debitterizing method is to put the kernels into water at $30-70{ }^{\circ} \mathrm{C}$ for several hours or days. However, in the process of debitterizing, many components are transferred to the debitterizing water, such as proteins, amygdalin and phenols, thus causing the water to become dark brown and leading to environmental pollution if discharged without any treatment $[10,11]$. So, the emission of this polluted water is severely prohibited by the environmental protection sectors in China.

Recently, great attention has been paid to these issues, including exploring some novel rapid and green debitterizing techniques [12,13], and analysis of the components and functional evaluation of 
the debitterizing water in order to decrease the production of debitterizing wastewater and promote the added-value of apricot kernel processing [11]. In addition, some manufacturers also concentrated the debitterizing water so as to accumulate the compounds included and reduce its pollution of the environment. However, no further performance has been conducted on the concentrated substances (solid sample) at present, which greatly restricts utilization and the development of products with these solids.

According to our preliminary studies, amygdalin is the most abundant active substance in the debitterizing wastewater, so it is very necessary to recycle these components for further utilization [11]. Many techniques have been reported to extract, purify or concentrate the functional compounds from natural products, among which adsorption by macroporous resins have been considered as the best method due to their high efficiency, low cost, favorable physicochemical stability, higher adsorption capacity, good selectivity, fast adsorption rate and convenient regeneration process [14,15]. The adoption of this method over other alternatives is mainly dependent on the factors relative to the adsorption, including cost, adsorption capacity for the particular adsorbate, regeneration and durability. In the meantime, the adsorbent's adsorption capacity can also be influenced by its similar polarity with the targeted compounds, higher surface area and larger average pore diameter [15-17]. As a novel method, no research has been conducted on the macroporous resins' application to the recycling of amygdalin from the concentrated debitterizing water of apricot kernels.

Consequently, the aim of this work was firstly to select the appropriate absorbent from the six different macroporous resins for purifying the amygdalin according to their adsorption behaviours, then the investigations were conducted on the adsorption/de-adsorption kinetics, and the thermodynamics for the static/dynamic adsorption of amygdalin in the debitterizing water. In addition, the isotherm models were determined for fitting the experimental data, and the values estimated for the enthalpy, free energy and entropy of the adsorption were also carried out for the selected XDA-1 absorbent resin. The main purpose of this study was to systematically study the feasibility of this method to purify the amygdalin from the concentrated debitterizing water with the XDA- 1 resin in order to reduce the loss of nutritional compounds, the emission of polluted water, and to promote the added value for apricot kernel processing.

\section{Materials and Methods}

\subsection{Samples and Preparation}

Samples of debitterizing water concentrate (DWC; dark brown solid) are the by-products from the debitterizing procedures of apricot kernel processing, and were kindly provided by the Shaanxi Tianshou Apricot Food Co. Ltd. (Xi'an City, China). The compositions and characteristics of the DWC were described as in the paper by Zhang et al. [11]. The sample of DWC was dissolved in water to prepare the solutions for use.

\subsection{Chemicals}

Standard amygdalin (purity over 98\%) was purchased from the Chengdu Perferred Bio-Technology Co. Ltd. (Chengdu City, China), methanol of high performance liquid chromatography (HPLC) purity was purchased from Thermo Fisher Scientific (Shanghai, China) Co. Ltd.

\subsection{Pre-Treatment of the Macroporous Resins}

Six kinds of commercial macroporous absorbent resins (XDA-1, ZGDM11, ZGDM130, H60, AB-8 and LSA-21) were tested to select the appropriate one for purifying the amygdalin from DWC, and their physical properties are shown in Table 1.

Before the experiments, the pre-treatment was conducted on the absorbents to remove the monomers inside the pores which were left as residue during the synthesis process, including 
soaking them in anhydrous ethanol for $24 \mathrm{~h}$, then washing successively with ethanol and deionized water, respectively.

Table 1. Physical properties of the six kinds of macroporous resins.

\begin{tabular}{cclcc}
\hline Model & Appearance & Polarity & Aperture $/ \mathbf{n m}$ & $\begin{array}{c}\text { Specific Surface } \\
\mathrm{Area} / \mathbf{m}^{\mathbf{2}} \cdot \mathbf{g}^{\mathbf{- 1}}\end{array}$ \\
\hline XDA-1 & Brown opaque globular particles & nonpolar & $8.5-9.5$ & $1000-1100$ \\
ZGDM11 & White translucent globular particles & nonpolar & $12.0-18.0$ & $\geq 800$ \\
H60 & White opaque globular particles & low-pole & $10.0-10.5$ & $540-580$ \\
ZGDM130 & White opaque globular particles & low-pole & $10.0-12.0$ & $450-550$ \\
AB-8 & White opaque globular particles & low-pole & $13.0-14.0$ & $480-520$ \\
LSA-21 & White opaque globular particles & semi-pole & 30 & $\geq 630$ \\
\hline
\end{tabular}

\subsection{Experiments of Static Adsorption/De-Adsorption of Macroporous Resin}

Each of the pre-treated absorbents $(1.0000 \mathrm{~g})$ was put into a conical flask with a stopper and contacted with $15 \mathrm{~mL}$ of the DWC sample solution. Subsequently, the flasks were shaken $(90 \mathrm{r} / \mathrm{min}$ ) in a thermostatic oscillator at $25^{\circ} \mathrm{C}$ for $12 \mathrm{~h}$ until equilibrium was achieved. Consequently, aliquots of solution were withdrawn to determine the amounts of amygdalin adsorbed by the macroporous resins.

The equilibrium-achieved resins were firstly rinsed with deionized water to remove the amygdalin on their surface, then carefully eluted by the $90 \%$ ethanol solution, and the concentration of amygdalin in the eluent was finally determined. The parameters like adsorption capacity, adsorption ratio and de-adsorption ratio were calculated as follows [18]:

$$
\begin{gathered}
Q(m g / g)=\left[\left[C_{0}-C_{e}\right] \times V_{a}\right] / W \\
A \%=\left[\left[C_{0}-C_{e}\right] / C_{0}\right] \times 100 \% \\
D \%=C_{d} V_{d} /\left[\left[C_{0}-C_{e}\right] \times V_{a}\right] \times 100 \%
\end{gathered}
$$

where $Q, A$ and $D$ represent the adsorption capacity $(\mathrm{mg} / \mathrm{g})$, adsorption ratio $(\%)$ and de-adsorption ratio (\%), respectively; $C_{0}$ is the concentration of amygdalin before adsorption $(\mathrm{mg} / \mathrm{g}), C_{e}$ is the concentration of amygdalin after adsorption equilibrium $(\mathrm{mg} / \mathrm{g}), \mathrm{C}_{\mathrm{d}}$ is the concentration of amygdalin in eluent $(\mathrm{mg} / \mathrm{g}) ; V_{a}$ and $V_{d}$ represent the volume $(\mathrm{mL})$ of DWC solution contacted with resins and effluent volume $(\mathrm{mL})$, respectively; and $\mathrm{W}$ is the resin weight $(\mathrm{g})$.

\subsection{Statics Adsorption Kinetics}

The adsorption kinetics curve of amygdalin on XDA-1, ZGDM11 and AB-8 were studied according to the following procedure: each of the pre-treated resins $(0.5 \mathrm{~g})$ was put into a conical flask with the stopper and contacted with $100 \mathrm{~mL}$ of DWC solution. Subsequently, the flasks were continually shaken in a thermostatic oscillatorat $25^{\circ} \mathrm{C}$, and aliquots of DWC solution were withdrawn at different time intervals to calculate the amounts of solute adsorbed by the resins and residuals in the solution until equilibrium was achieved.

\subsection{Static Adsorption Isotherm Procedure}

Adsorption isotherms were performed according to the following procedures: each of the pre-treated XDA-1 resins (fixed weight) was put into a conical flask, which contained the DWC solutions (fixed volume) of different concentrations including $63.63 \mathrm{mg} / \mathrm{mL}, 50.91 \mathrm{mg} / \mathrm{mL}, 38.18 \mathrm{mg} / \mathrm{mL}$, $25.45 \mathrm{mg} / \mathrm{mL}$ and $16.05 \mathrm{mg} / \mathrm{mL}$, respectively. All of these values of the concentrations were obtained from the preliminary experiments about the different diluted solutions in our laboratory. The flasks were placed in a thermostatic water bath at three temperatures $\left(25^{\circ} \mathrm{C}, 35^{\circ} \mathrm{C}\right.$ and $\left.45^{\circ} \mathrm{C}\right)$ and shaken for several hours until equilibrium was achieved; the higher the temperature, the shorter the equilibration 
time. The content of the amygdalin in the DWC solutions was determined by the high performance liquid chromatography (HPLC).

In order to select a suitable model to describe the adsorption properties of the XDA- 1 resin, the classic Freundlich model were used to fit the experimental results for the adsorption of amygdalin:

$$
\ln Q_{e}=1 / n \ln C_{e}+\ln K_{f}
$$

where $Q_{e}$ is the equilibrium adsorption capacity $(\mathrm{mg} / \mathrm{g}), C_{e}$ is the equilibrium concentration $(\mathrm{mg} / \mathrm{g})$, $n$ is the characteristic constant, and $\mathrm{K}_{\mathrm{f}}$ is the equilibrium adsorption constant.

\subsection{Effect of Ethanol Concentration on the De-Adsorption of Amygdalin for the XDA-1 Resin}

$0.5 \mathrm{~g}$ of XAD-1 resin was put into the DWC solutions in a conical flask with a stopper, then the flask was fixed in a thermostatic water bath at $25^{\circ} \mathrm{C}$ and shaken for $6 \mathrm{~h}$ to achieve equilibrium. The concentration of the amygdalin remaining in the DWC solutions was determined to calculate the equilibrium adsorption capacity of the XAD-1 resin. Consequently, the XAD-1 resins equilibrated with the amygdalin were washed with the deionized water, put into the triangle bottles filled with $15 \mathrm{~mL}$ of $20 \%, 40 \%, 60 \%, 80 \%$, and $100 \%$ ethanol solution and kept shaken in a water thermostatic bath for $24 \mathrm{~h}$, respectively. Finally, the concentration of the amygdalin in each of the ethanol solutions was determined to compare the effect of ethanol concentrations on the de-adsorption of amygdalin for the XAD-1 resin.

\subsection{Effect of Time on the De-Adsorption Capacity of XAD-1 for the Amygdalin}

$0.5 \mathrm{~g}$ of XAD-1 resins were added to the DWC solutions in a flask, which was shaken in a thermostatic water bath at $25^{\circ} \mathrm{C}$ for $6 \mathrm{~h}$ to achieve the equilibrium. The resins were then washed with the deionized water and de-absorbed with $80 \%$ ethanol solution, and the de-adsorption capacity for the amygdalin was continuously measured for $6 \mathrm{~h}$ at different time intervals, and the curve of the static de-adsorption volume against time was drawn.

\subsection{Purification of Amygdalin in DWC Solutions by the XAD-1 Resin}

The XDA-1 resin was soaked in ethanol, then the cleaned resin was continuously packed into the glass column and rinsed with deionized water for further use.

\subsubsection{Dynamic Adsorption of XDA-1 Resin on Amygdalin}

The dynamic adsorption experiments were conducted as follows: the DWC solutions of $78.05 \mathrm{mg} / \mathrm{mL}$ and $35.70 \mathrm{mg} / \mathrm{mL}$ were loaded to the packed column with XDA-1 resin at the flow rate of $2 \mathrm{~mL} / \mathrm{min}$, respectively, and the eluents were collected into a tube with every $10 \mathrm{~mL}$ by the auto-fraction collector (ShanghaiJiapeng Technology Co. Ltd., Shanghai, China). Finally, the determination was conducted on the amygdalin concentration in the elutions, when the concentration is $10 \%$ or less of the initial concentration, the adsorption equilibrium was considered to have been achieved. The dynamic adsorption curve was plotted according the data obtained.

\subsubsection{Dynamic De-Adsorption of XDA-1 Resin on Amygdalin}

The dynamic de-adsorption experiments were obtained as follows: a certain volume of DWC solution $(78.05 \mathrm{mg} / \mathrm{mL})$ was firstly loaded onto the glass column packed with XDA-1 resin, before de-adsorption with $80 \%$ ethanol solution, the XDA-1 resin was washed with deionized water to remove the non-targeted compounds. Finally, the eluted solution was collected using an automatic collector $(8 \mathrm{~mL} /$ tube) and determined until no amygdalin was included in the eluted solution. The dynamic de-adsorption curve of XDA-1 resin on amygdalin was plotted according the data obtained. 


\subsubsection{Determination of the Recovery Rate and Relative Content of Amygdalin}

As mentioned in Section 2.9.2, all the collected elutions were mixed together and the amygdalin content was determined. Consequently, the amygdalin recovery rate and the relative content (peak area normalization method) were calculated by the following formula:

$$
\begin{gathered}
Y(\%)=C_{1} M_{1} / C M \times 100 \% \\
W=A_{0} / A_{i} \times 100 \%
\end{gathered}
$$

where $\mathrm{Y}$ is the recovery rate of amygdalin (\%); $C$ is the concentration of amygdalin in the sample before purification; $C_{1}$ is the concentration of amygdalin in the elution after purification; $M$ is the loaded volume; $M_{1}$ is the eluted volume; $W$ is the relative content of amygdalin (\%); $A_{0}$ is the peak area of amygdalin; $A_{i}$ is the total peak areas in the chromatograms.

\subsection{Determination of Amygdalin by HPLC}

The amygdalin content was determined by HPLC (Dalian Elite Analytical Instrument Co. Ltd., Dalian City, China) equipped with a P230II binary pump, a Rheodyne injector (loop, $20 \mu \mathrm{L}$ ) and a UV230II detector (Elite) according to the method reported by our group [11]. Chromatograms were recorded by the EC2006 software (Elite). Samples were separated on a column of TC-C $18(5.0 \mu \mathrm{m}$, $4.6 \mathrm{~mm} \times 250 \mathrm{~mm}$, Agilent, Palo Alto City, CA, USA). All mobile phases were ultrasonically degassed for $25 \mathrm{~min}$ and filtered through a $0.45 \mu \mathrm{m}$ membrane prior to use. An isocratic elution method was applied for the separation of amydalin with the mobile phases of Methanol- $\mathrm{H}_{2} \mathrm{O}(28: 72 ; v / v), 1.0 \mathrm{~mL} / \mathrm{min}$ of flow rate, $35{ }^{\circ} \mathrm{C}$ of column temperature. The detection wavelength was set at $214 \mathrm{~nm}$, and the injection volume was $20 \mu \mathrm{L}$. Each sample was performed in triplicate.

\section{Results and Discussion}

\subsection{Selection of Resins Suitable for Adsorption/De-Adsorption of Amygdalin}

The adsorption and de-adsorption capacity of the macroporous resins for the amygdalin are shown in Figure 1. It is obvious that the adsorption capacities of the XDA-1, ZGDM11, AB-8 and ZGDM130 resins were higher than those of the LSA-21 and H60 resins. Although the resin of ZGDM130 had the higher adsorption capacity, its de-adsorption capacity was lower. Considering all of these factors, the resins of ZGDM130, LSA-21 and H60 were thought not suitable for the purification of the amygdalin in this research. Their different adsorption and de-adsorption capacities can be explained by their characteristic properties, such as polarity and specific surface. Based on the adsorption and de-adsorption capacity, the resins of XDA-1, ZGDM11 and AB-8 were screened for further study.

\subsection{Comparison of Static Adsorption Kinetics for Three Resins on the Amygdalin}

As shown in Figure 2, all the above-screened three resins achieved the adsorption equilibrium within $2 \mathrm{~h}$, although their equilibrium adsorption capacities were different. In the beginning of the adsorption, a similar rapid increase of adsorption rate happened on the three resins, which may be due to the small pore size of the selected macroporous resins. Moreover, the amygdalin is a small molecule with a molecular weight of 457 Dalton, so it can rapidly diffuse into the pores of the resins. The following slight increase in the adsorption rate may be attributed to the reduced concentration of the amygdalin in the sample solution or the adsorption saturation phenomenon on the resin's surface. In comparison, the XDA-1 resin had a higher adsorption capacity than that of the ZGDM11 and AB-8 at the adsorption equilibrium state, that is, the XDA-1 resin had the good affinity for the amygdalin, so it was screened to purify the amygdalin from the sample of DWC in the following experiments. 


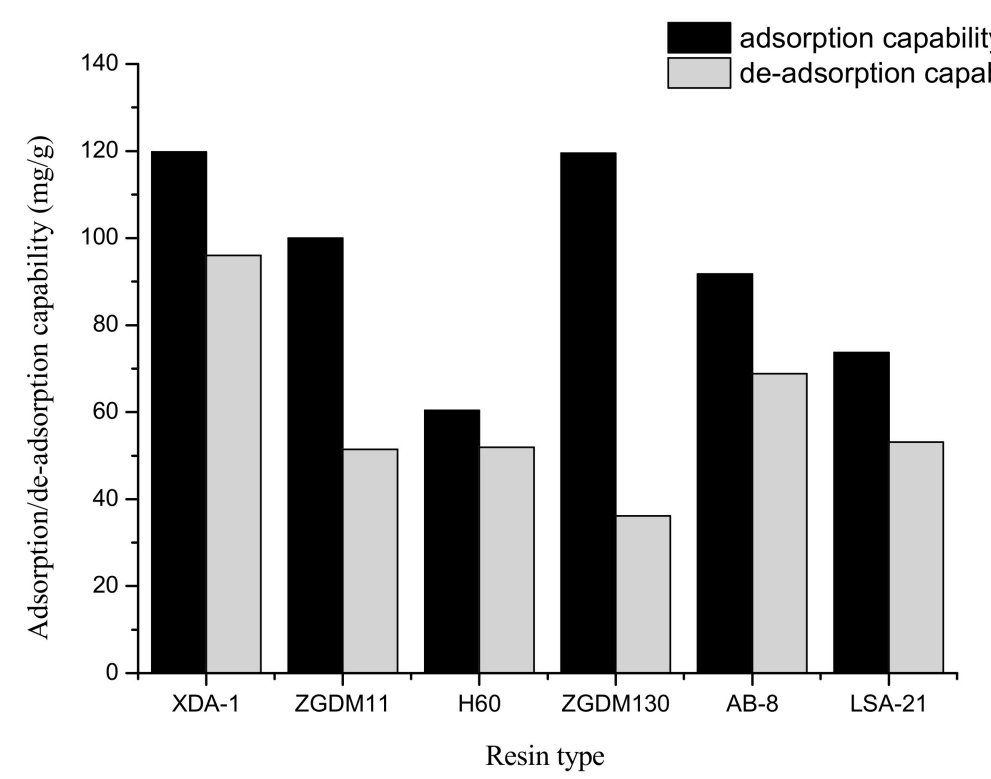

Figure 1. Adsorption and de-adsorption capacity of the six kinds of macroporous resins for the amygdalin.

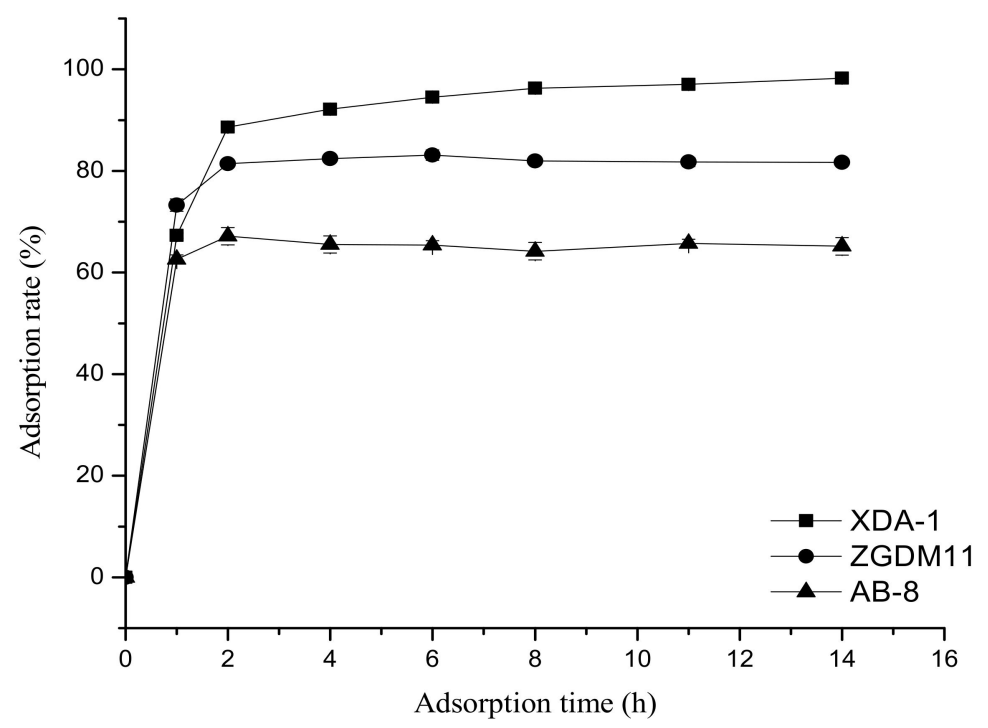

Figure 2. Static adsorption kinetics of the three macroporous resins.

\subsection{Static Adsorption Isotherm of XDA-1 Resin on the Amygdalin}

The adsorption isotherm of XDA-1 resin on the amygdalin was investigated at three different temperatures of $25^{\circ} \mathrm{C}, 35^{\circ} \mathrm{C}$ and $45^{\circ} \mathrm{C}$, and the results were shown in Figure 3 . For the same equilibrium concentration, a decreasing adsorption capacity is very obvious with the increasing temperature, and the curves corresponding to the higher temperatures lay below the ones corresponding to the lower temperatures. On the whole, the temperature was negatively correlated with the adsorption capacity, that is, increasing the temperature was unfavorable to the adsorption process, which suggests that the XDA-1 resin's adsorption on the amygdalin was an exothermic process and controlled by physical mechanisms [19]. In addition, the slightly convex upward isotherm curve belongs to the preferential adsorption type. 


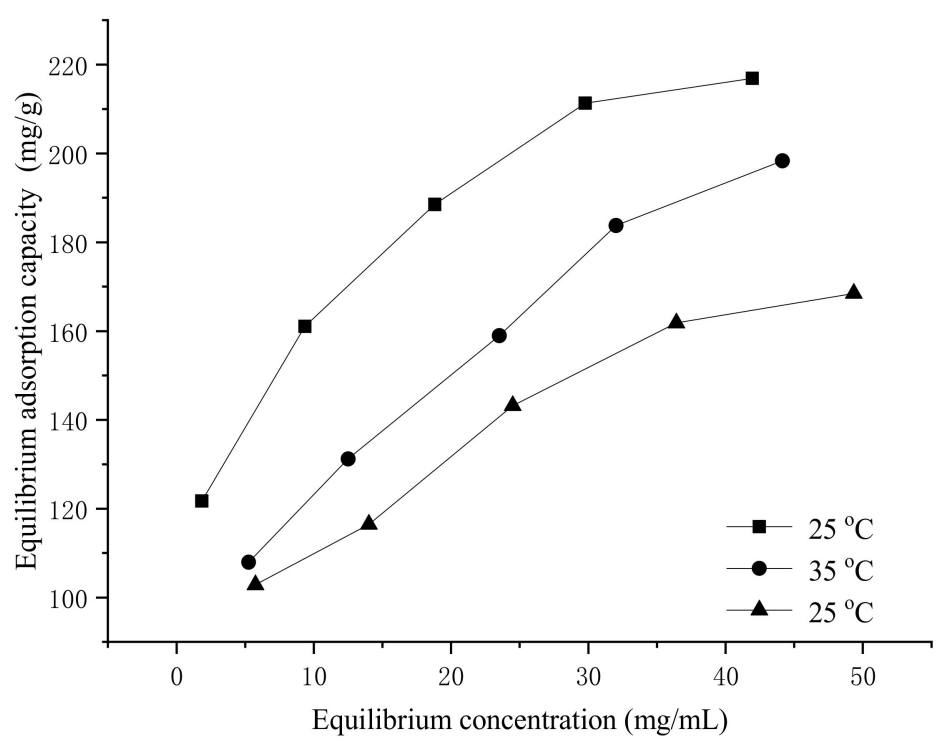

Figure 3. Adsorption isotherm of XDA-1 resin on the amygdalin.

The isotherms were usually used to describe the relations between the equilibrium concentration and the equilibrium adsorption capacity at the same temperature. The Freundlich model is usually considered the most popular model for describing the equilibrium adsorption isotherm by comparing correlation coefficients [20,21]; the relevant data were mapped and analyzed in Excel, and the results are shown in Figure 4 and Table 2.

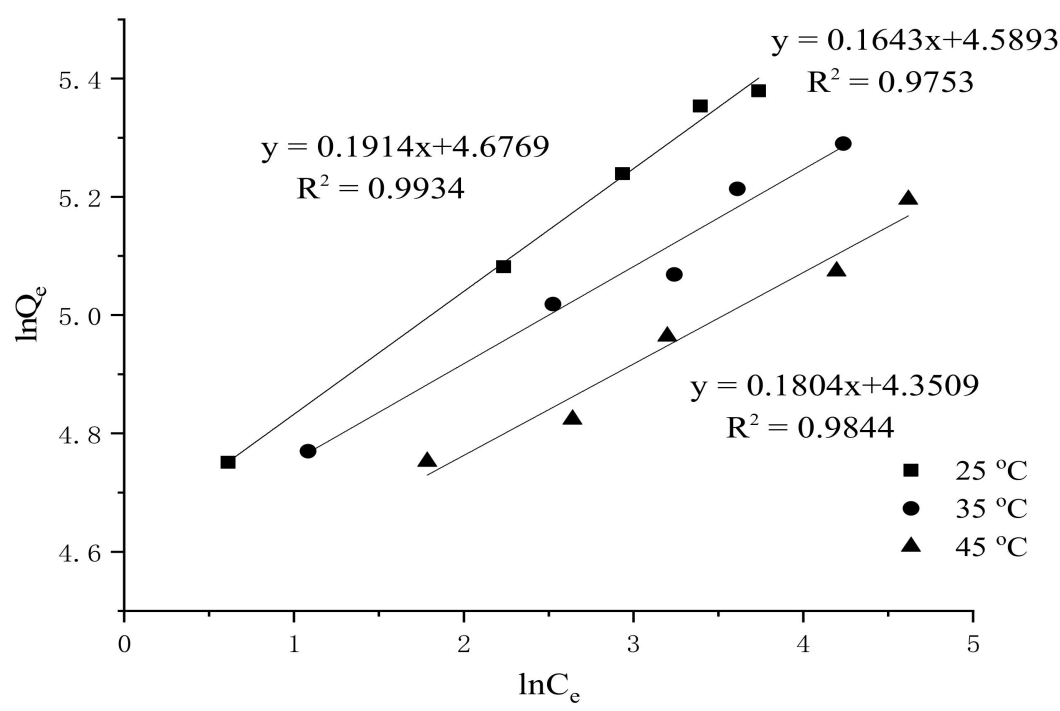

Figure 4. Linear relationship between $\ln Q_{e}$ and $\ln C_{e}$.

Table 2. Fitted Freundlich equations and parameters.

\begin{tabular}{ccccc}
\hline T/K & Fitted Equation & $\operatorname{lnK}_{\mathbf{f}}$ & $\mathbf{n}$ & $\mathbf{R}^{\mathbf{2}}$ \\
\hline 298 & $\ln \mathrm{Q}_{\mathrm{e}}=0.1914 \ln C_{\mathrm{e}}+4.6769$ & 4.677 & 5.225 & 0.9934 \\
308 & $\ln \mathrm{Q}_{\mathrm{e}}=0.1643 \ln C_{\mathrm{e}}+4.5893$ & 4.589 & 6.086 & 0.9753 \\
318 & $\ln \mathrm{Q}_{\mathrm{e}}=0.1804 \ln \mathrm{C}_{\mathrm{e}}+4.3509$ & 4.351 & 5.544 & 0.9844 \\
\hline
\end{tabular}

The correlation coefficients for the Freundlich model on the XDA-1 resin's adsorption of amygdalin at different temperatures are listed in Table 2. The correlation coefficient of the regression equation is more than 0.97 , implying that the Freundlich equation is a better model for describing the equilibrium adsorption isotherm. The parameter of $\mathrm{n}$ in the Freundlich model represents the adsorption performance 
of the resin, and the values of $\mathrm{n}$ ranged from 1 to 10, which can demonstrate the resin's better adsorption performance [15]. Moreover, the higher value of $\mathrm{n}$ than 1 indicates that the XDA-1 resin can be applied to purify the amygdalin from the DWC sample.

\subsection{Thermodynamics Adsorption of Amygdalin by the XDA-1 Resin}

Enthalpy is a function of state, and the value of enthalpy can predict the type of the adsorption process, that is, endothermic or exothermic. Enthalpy changes $(\Delta \mathrm{H})$ can be calculated by the Clausius-Clapeyron equation as follows:

$$
\ln C_{e}=\Delta H / R T+K
$$

where $C_{e}$ is the concentration corresponding to the adsorption capacity of $Q_{e}(\mathrm{mg} / \mathrm{g}), T$ is the thermodynamic temperature $(\mathrm{K}), \mathrm{R}$ is the ideal gas constant $\left(8.314 \mathrm{~J} \cdot \mathrm{mol}^{-1} \cdot \mathrm{K}^{-1}\right), \Delta H$ is the equivalent adsorption enthalpy $\left(\mathrm{kJ} \cdot \mathrm{mol}^{-1}\right), \mathrm{K}$ is the constant.

Values for the free energy of adsorption can be obtained using an expression derived from the Gibbs adsorption isotherm:

$$
\Delta G=-n R T
$$

where $n$ is the correlation coefficient of the Freundlich equation.

Entropy variation values $(\Delta S)$ can be estimated using the previously calculated $\Delta H$ and $\Delta G$ values and the Gibbs-Helmholtz equation:

$$
\Delta S=(\Delta H-\Delta G) / T
$$

The adsorption isometric line was made up of the isotherms at different temperatures from Figure 5, and the $\Delta H$ could be calculated from the slope of the plot of $\operatorname{lnCe}$ as a function of $1 / T$ [22]. The results are shown in Figure 5 and Table 3.

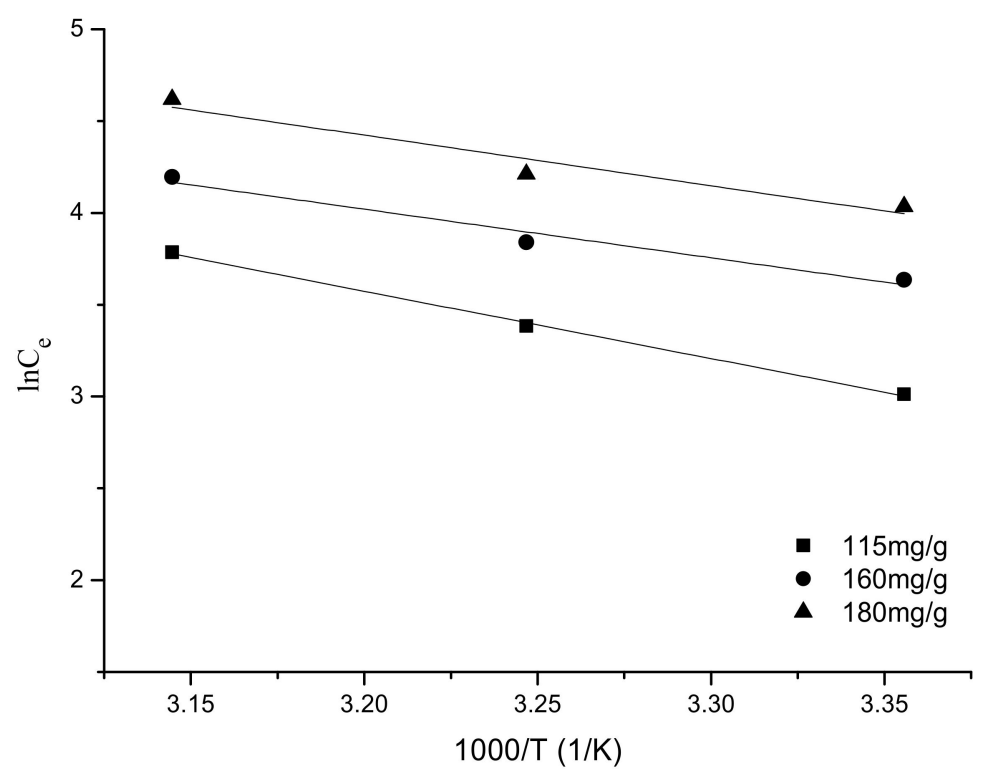

\begin{tabular}{|c|c|c|c|c|}
\hline \multirow{2}{*}{$Q_{e}(m g / g)$} & \multirow{2}{*}{$\Delta \mathrm{H}(\mathrm{kJ} / \mathrm{mol})$} & \multicolumn{3}{|c|}{$\Delta \mathrm{S}\left(\mathrm{kJ} \cdot \mathrm{mol}^{-1} \cdot \mathrm{K}^{-1}\right)$} \\
\hline & & $298 \mathrm{~K}$ & $308 \mathrm{~K}$ & $318 \mathrm{~K}$ \\
\hline 115 & -4.6017 & 0.0361 & 0.0326 & 0.0262 \\
\hline 160 & -5.4799 & 0.0331 & 0.0298 & 0.0235 \\
\hline 180 & -5.6264 & 0.0326 & 0.0293 & 0.0230 \\
\hline
\end{tabular}

Figure 5. Linear relationship between $\ln Q_{e}$ and $1 / T$.

Table 3. Thermodynamic parameters for the adsorption of amygdalin by XDA-1 resin. 
As shown in Table 3, the enthalpy changes are always negative, which indicates an exothermic process. In general, increasing the temperature was unfavorable to the adsorption process [23]. The absolute value of $\Delta \mathrm{H}$ for the adsorption process of XDA-1 resin on the amygdalin was less than $12 \mathrm{~kJ} / \mathrm{mol}$, indicating that the adsorption of amygdalin onto the XDA-1 resin's surface was controlled by physical mechanisms [19], that is, it is a physical adsorption process. The absolute value of $\Delta \mathrm{H}$ increased with the increasing $Q_{e}$ value, which might be the direct contact between the adsorbents and the target substances; when the amygdalin concentration was lower, it could occupy a favorable adsorption position due to the uneven distribution of the resin.

The free adsorption energy $(\Delta \mathrm{G})$ of XDA- 1 on the amygdalin at the temperatures of $25^{\circ} \mathrm{C}, 35^{\circ} \mathrm{C}$ and $45{ }^{\circ} \mathrm{C}$ were $-15.352,-14.655$ and $-12.945 \mathrm{~kJ} / \mathrm{mol}$, respectively. All of these negative values indicate that the amygdalin was more likely to migrate from the adsorption solution to the resin's surface, and the process was spontaneous. As the absolute value of $\Delta \mathrm{G}$ decreased with the increasing temperature, the spontaneous tendency of the adsorption process decreased, which confirms that the adsorption is a physical adsorption process [24].

The positive value of $\Delta S$ indicates that the molecules in the solid/ liquid two-phase interface are in a state of chaotic movement during the adsorption process. Generally, the resin adsorbs the amygdalin, and a close and ordered arrangement of the water molecules will be excluded at the same time, since the amygdalin molecule was larger than that of the water molecules, and each amygdalin molecule adsorption would lead to the multiple water molecules being desorpted, and thus the overall chaos of the system leads to an increase in entropy.

\subsection{Effect of Ethanol Concentration on the De-Adsorption of Amygdalin for the XDA-1 Resin}

Figure 6 reveals that the ethanol concentration was significant for the de-adsorption of the amygdalin from the XDA-1 resin. At a range from $20 \%$ to $80 \%$, the de-adsorption ratios increased with the increasing of ethanol concentration; when the concentration surpassed $80 \%$, the de-adsorption ratios decreased. The de-adsorption ratio reached its highest value with the ethanol concentration at $80 \%$. The essence of the de-adsorption process is the competitive ad-adsorption of eluent and adsorbent to the target. On the whole, the ethanol concentration of $80 \%$ is considered to be the optimum eluent for the XDA-1 resin to de-adsorb the amygdalin.

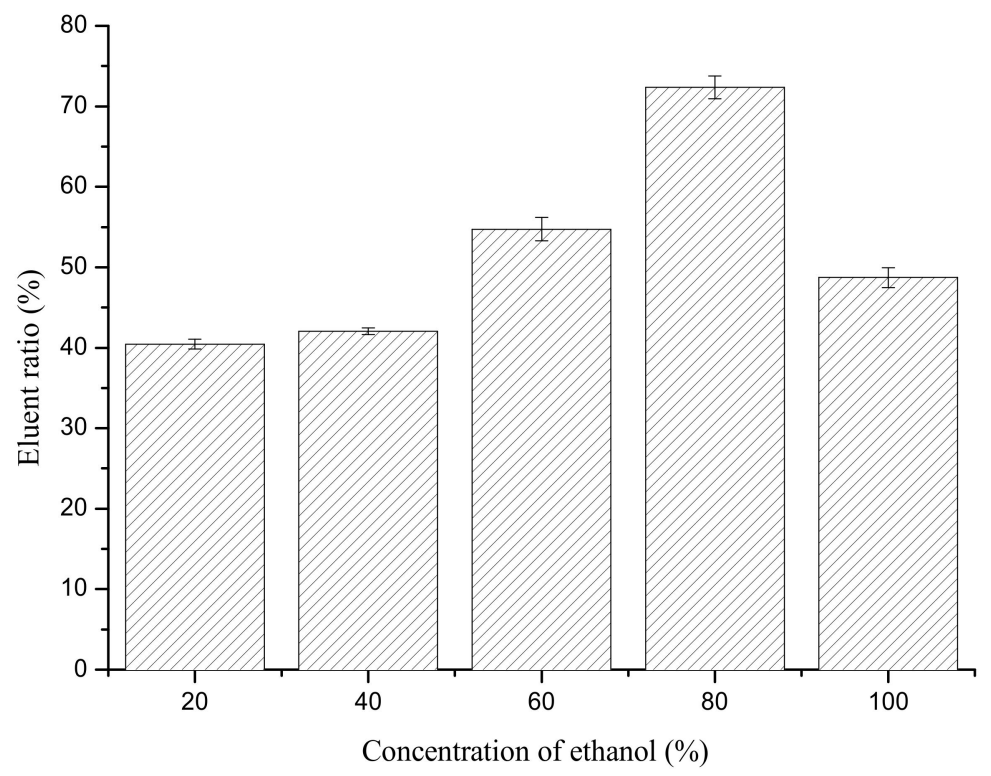

Figure 6. Effect of ethanol concentration on the de adsorption ratio of amygdalin from the XDA-1 resin. 


\subsection{Static Elution Curve of Amygdalin from the XDA-1 Resin}

As can be seen in Figure 7, the de-adsorption amount of amygdalin increased with the de-adsorption time increasing, and the value reached maximum with the time to $4.5 \mathrm{~h}$, followed by a slight decrease. Therefore, the optimum static de-adsorption time was $4.5 \mathrm{~h}$, when the $80 \%$ ethanol solution was used for the de-adsorption of amygdalin from the XDA-1 resin.

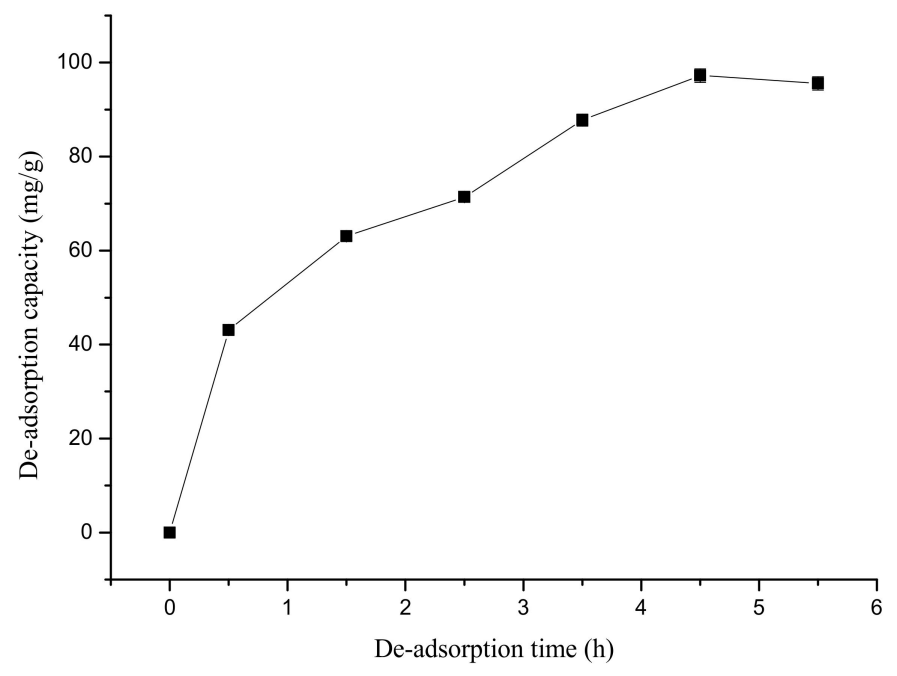

Figure 7. Effect of time on the de-adsorption capacity of amygdalin from the XDA-1 resin.

\subsection{Dynamic Adsorption and De-Adsorption Experiments}

\subsubsection{Effect of Sample Concentration on the Adsorption of Amygdalin for the XDA-1 Resin}

The DWC solutions to be loaded were fixed at $78.05 \mathrm{mg} / \mathrm{mL}$ and $35.70 \mathrm{mg} / \mathrm{mL}$, respectively. As shown in Figure 8, it took longer to reach the adsorption equilibrium point when the sample concentration was at $35.70 \mathrm{mg} / \mathrm{mL}$. The saturation point (i.e., the concentration of the amygdalin in the effluent was about $10 \%$ of the initial concentration of the sample solution) was reached when the sample volume was up to $180 \mathrm{~mL}$. While for the sample concentration of $78.05 \mathrm{mg} / \mathrm{mL}$, the volume reaching the saturation point was about $120 \mathrm{~mL}$. Generally, increasing the loading concentration had a negative effect on achieving the adsorption equilibrium. If the concentration was too low, the practical operation would consume longer time and occupy more resources. Therefore, the optimum loading concentration was selected to be $78.05 \mathrm{mg} / \mathrm{mL}$ for the adsorption of amygdalin with the XDA- 1 resin.

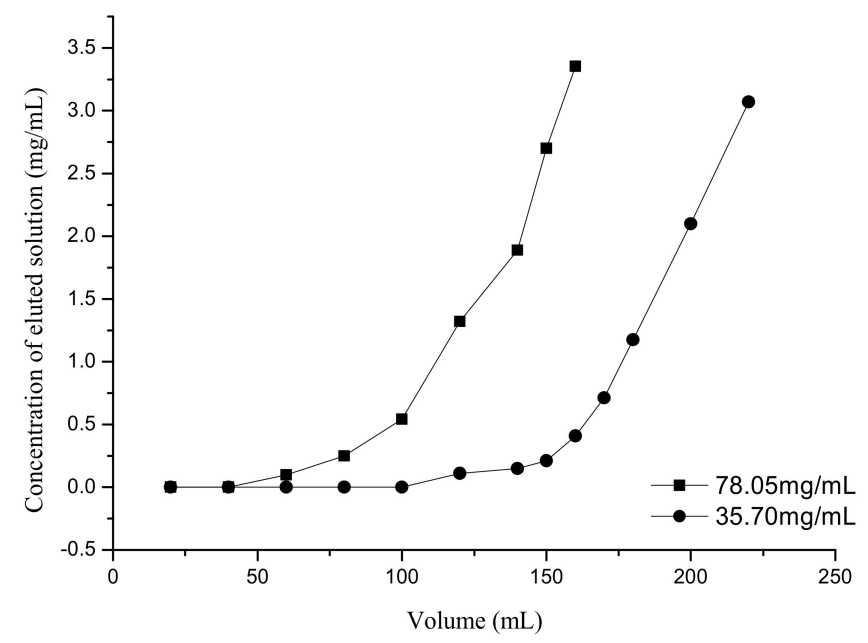

Figure 8. Effect of different sample concentration on adsorption of amygdalin with XDA-1 resin. 


\subsubsection{Dynamic De-Adsorption Curve of Amygdalin with XDA-1 Resin}

After the adsorption was equilibrated on the column packed with XDA-1 resin, the washing was conducted with the deionized water to remove the proteins, polysaccharides and so on. Consequently, the column was eluted with the $80 \%$ ethanol solution, the eluents were collected using an automatic collector, and the amygdalin was determined for all of the tubes collected. The dynamic de-adsorption curve is shown in Figure 9, it can be seen that the amygdalin appeared within the elution time of 40 80 $\mathrm{min}$, and the eluents collected within this period were mixed for rotary evaporation.

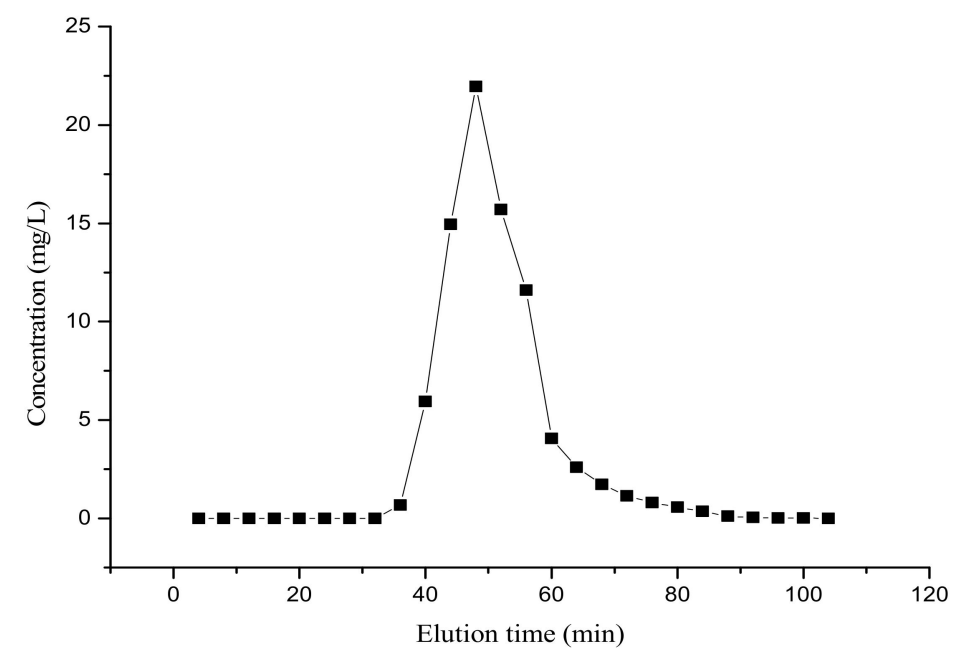

Figure 9. Dynamic de-adsorption curve of XDA-1 resin for the amygdalin.

\subsection{Effect of Purification of XDA-1 Resin on the Amygdalin}

Figures 10 and 11 show the full wavelength scanning and the HPLC chromatograms for the DWC samples before and after purification with the XDA-1 resin, respectively. It could be obviously seen that the impurities in the DWC solutions significantly reduced after purification, while the amygdalin changed a little, indicating that the XDA- 1 resin is suitable for the purification of amygdalin. The recovery of the amygdalin in DWC was $88.75 \%$, and the relative content of amygdalin in DWC increased from $34.04 \%$ to $61.58 \%$ according to the changes of peak areas in the HPLC chromatograms.

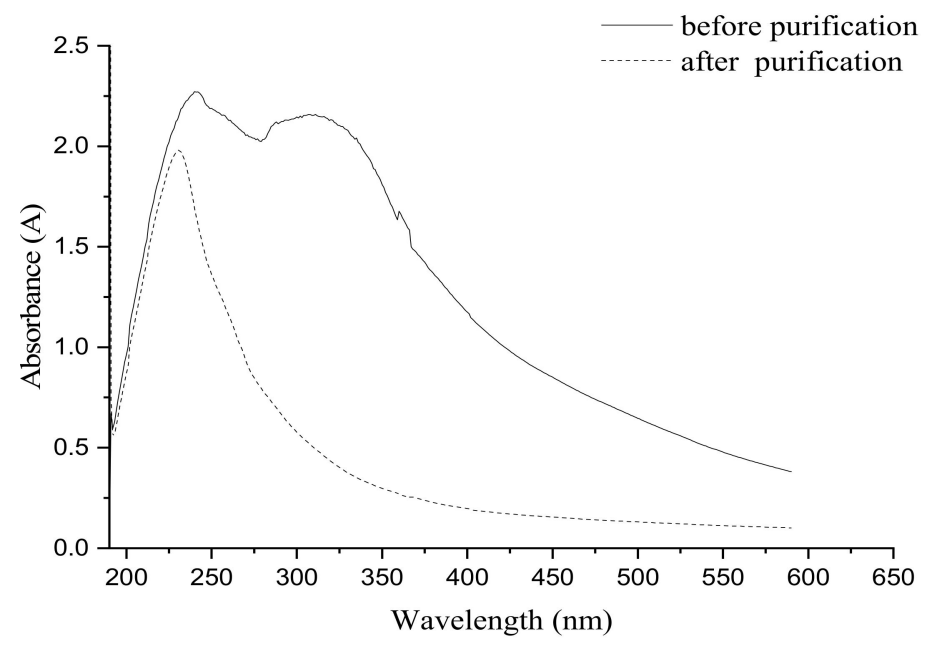

Figure 10. Full wavelength scanning of samples before and after purification by XDA-1 resin. 

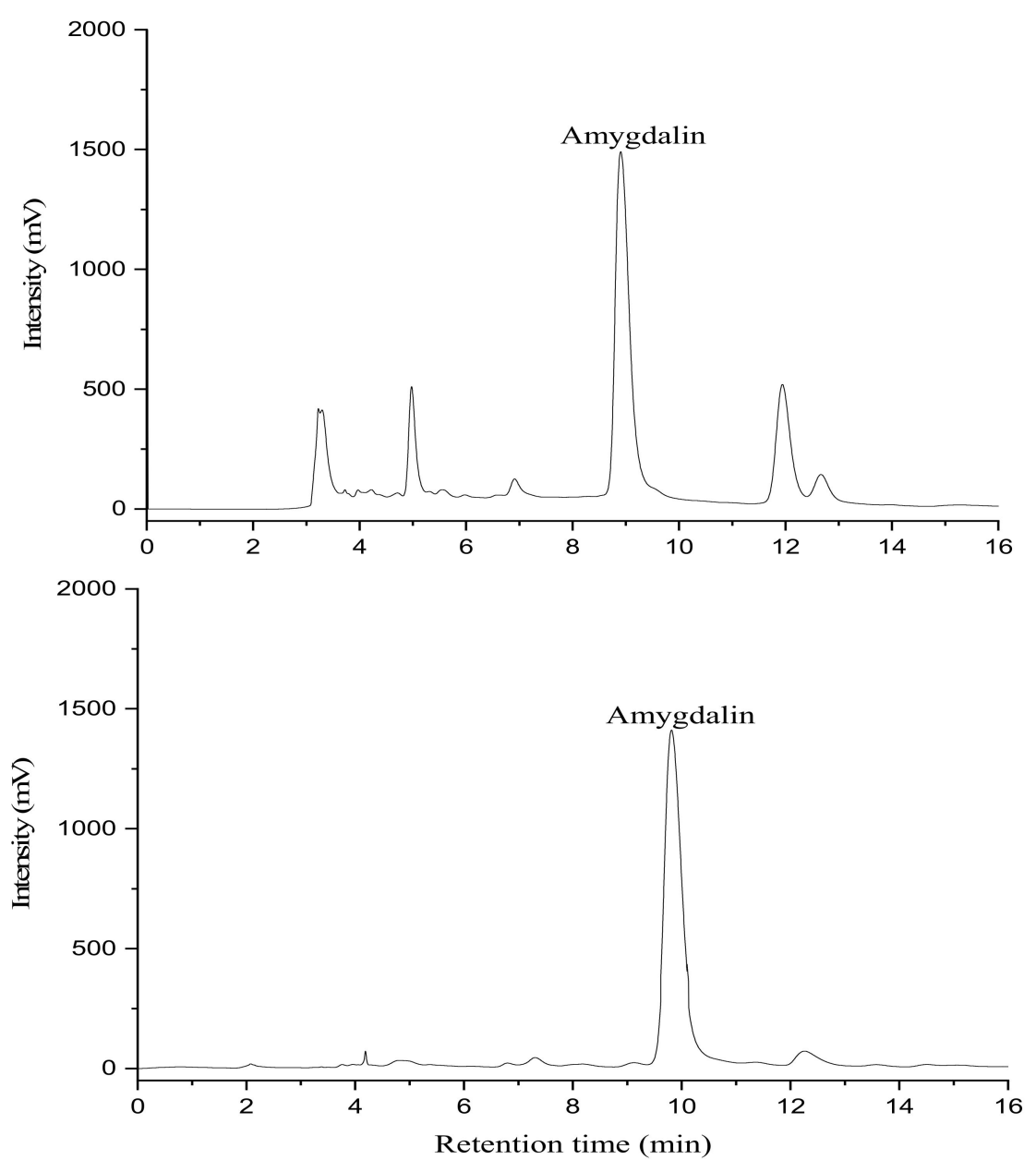

Figure 11. High performance liquid chromatography (HPLC) chromatograms of debitterizing wastewater concentrate (DWC) samples before and after purification.

\section{Conclusions}

According to the data obtained in this study, we can confirm that the XDA-1 resin is suitable for the purification of amygdalin from the DWC sample, the wastewater concentrate from the debitterizing of apricot kernels. With one run treatment by XDA- 1 resin, the recovery rate achieved was $88.75 \%$ and the relative content increased from $34.04 \%$ to $61.58 \%$. These results indicate that the wastewater generated from the debitterizing of apricot kernels can be well treated, on one hand to reduce its pollution to environment and on the other hand to recycle the bioactive compounds, hence promoting added-value for apricot kernels in the processing industry. To the best of our knowledge, no similar works have been conducted on these by-products until now. However, the development of products using the recycled amygdalin should be further studied, and the possibility of simpler pre-treatment (without pre-concentrating) of the wastewater should also be investigated.

Author Contributions: In this research, all authors contributed effectively. C.-X.W. designed, achieved experiments and performed research; D.-D.W. analyzed the data, performed data interpretation, and wrote the paper; Q.-A.Z. supervised the project and revised the manuscript.

Funding: This study was funded by National Natural Science Foundation of China [No. 31101324], Key Research Development Program of Shaanxi Province, China [No. 2018ZDXM-NY-086] and the Fundamental Research Funds for the Central Universities of China [No. GK201602005].

Conflicts of Interest: Author of Q.-A.Z. has received research grants from the above-mentioned organizations, and declares that he has no conflict of interest. D.-D.W. declares that she has no conflict of interest. C.-X.W. declares that she has no conflict of interest. 


\section{References}

1. Erdogan-Orhan, I.; Kartal, M. Insights into research on phytochemistry and biological activities of Prunus armeniaca L. (apricot). Food Res. Int. 2011, 44, 1238-1243. [CrossRef]

2. Górnaś, P.; Siger, A.; Seglina, D. Physicochemical characteristics of the cold-pressed Japanese quince seed oil: New promising unconventional bio-oil from by-products for the pharmaceutical and cosmetic industry. Ind. Crops Prod. 2013, 48, 178-182. [CrossRef]

3. Femenia, A.; Rossello, C.; Mulet, A.; Canellas, J. Chemical composition of bitter and sweet apricot kernels. J. Agric. Food Chem. 1995, 43, 356-361. [CrossRef]

4. Turan, S.; Topcu, A.; Karabulut, I.; Vural, H.; Hayaloglu, A.A. Fatty acid, triacylglycerol, phytosterol, and tocopherol variations in kernel oil of Malatya apricots from Turkey. J. Agric. Food Chem. 2007, 55, 10787-10794. [CrossRef] [PubMed]

5. Sharma, R.; Gupta, A.; Abrol, G.S.; Joshi, V.K. Value addition of wild apricot fruits grown in North-West Himalayan regions-a review. J. Food Sci. Technol. 2014, 51, 2917-2924. [CrossRef] [PubMed]

6. Özcan, M. Composition of some apricot (Prunus armeniaca L.) kernels grown in Turkey. Acta Aliment. Hung. 2000, 29, 289-294. [CrossRef]

7. Blaheta, R.A.; Nelson, K.; Haferkamp, A.; Juengel, E. Amygdalin, quackery or cure? Phytomedicine 2016, 23, 367-376. [CrossRef]

8. Wang, T.; Lu, S.-M.; Xia, Q.; Fang, Z.-X.; Johnson, S. Separation and purification of amygdalin from thinned bayberry kernels by macroporous adsorption resins. J. Chromatogr. B 2015, 975, 52-58. [CrossRef]

9. Zhang, Q.-A.; Song, Y.; Wang, X.; Zhao, W.-Q.; Fan, X.-H. Mathematical modeling of debittered apricot (Prunus armeniaca L.) kernels during thin-layer drying. CyTA J. Food 2016, 14, 509-517. [CrossRef]

10. Song, Y.; Zhang, Q.-A.; Fan, X.-H.; Zhang, X.-Y. Effect of debitterizing treatment on the quality of the apricot kernels in the industrial processing. J. Food Process. Pres. 2017, 42, 1-8. [CrossRef]

11. Zhang, Q.-A.; Wei, C.-X.; Fan, X.-H.; Shi, F.-F. Chemical compositions and antioxidant capacity of by-products generated during the apricot kernels processing. CyTA J. Food 2018, 16, 422-428. [CrossRef]

12. Zhang, Q.-A.; Fan, X.-H.; Zhang Yang, J.-N.; Zhang, Z.-Q. A Novel Method for Rapid Debitterizing of Apricot Kernels. Chinese Patent ZL 201310376132.X, 2016.

13. Fan, X.-H.; Zhang, Q.-A.; Liu, M.; Tian, C.-R. Progress in detoxification techniques of apricot kernels. Sci. Technol. Food Ind. 2014, 35, 396-399. (In Chinese)

14. Jia, G.-T.; Lu, X.-Y. Enrichment and purification of madecassoside and asiaticoside from Centella asiatica extracts with macroporous resins. J. Chromatogr. A 2008, 1193, 136-141. [CrossRef] [PubMed]

15. Xi, L.-S.; Mu, T.-H.; Sun, H.-N. Preparative purification of polyphenols from sweet potato (Ipomoea batatas L.) leaves by AB-8 macroporous resins. Food Chem. 2015, 172, 166-174. [CrossRef]

16. Idris, Z.M.; Dzahir, M.I.H.M.; Jamal, P.; Barkat, A.A.; Xian, R.L.W. Purification of bioactive phenolics from Phanerochaete chysosporium biomass extract on selected macroporous resins. IOP Con. Ser. Mater. Sci. Eng. 2017, 206, 012070. [CrossRef]

17. Liu, P.-W.; Du, Y.-F.; Zhang, X.-W.; Sheng, X.-N.; Shi, X.-W.; Zhao, C.-C.; Zhu, H.; Wang, N.; Wang, Q.; Zhang, L.-T. Rapid analysis of 27 components of Isodon serra by LC-ESI-MS-MS. Chromatographia 2010, 72, 265-273. [CrossRef]

18. Dong, Y.; Zhao, M.-M.; Sun-Waterhouse, D.-X.; Zhuang, M.-Z.; Chen, H.-P.; Feng, M.-Y.; Lin, L.-Z. Absorption and desorption behaviour of the flavonoids from Glycyrrhiza glabra L. leaf on macroporous adsorption resins. Food Chem. 2015, 168, 538-545. [CrossRef] [PubMed]

19. Gökmen, V.; Serpen, A. Equilibrium and kinetic studies on the adsorption of dark colored compounds from apple juice using adsorbent resin. J. Food Eng. 2002, 53, 221-227. [CrossRef]

20. Allen, S.J.; McKay, G.; Porter, J.F. Adsorption isotherm models for basic dye adsorption by peat in single and binary component systems. J. Colloid Int. Sci. 2004, 280, 322-333. [CrossRef]

21. Sohn, S.; Kim, D. Modification of Langmuir isotherm in solution systems-Definition and utilization of concentration dependent factor. Chemosphere 2005, 58, 115-123. [CrossRef]

22. Özcan, A.; Özcan, A.S.; Tunali, S.; Akar, T.; Kiran, I. Determination of the equilibrium, kinetic and thermodynamic parameters of adsorption of copper(ii) ions onto seeds of Capsicum annuum. J. Hazard. Mated. 2005, 124, 200-208. [CrossRef] [PubMed] 
23. Lin, L.-Z.; Zhao, H.-F.; Dong, Y.; Yang, B.; Zhao, M.-M. Macroporous resin purification behavior of phenolics and rosmarinic acid from Rabdosia serra (MAXIM.) HARA leaf. Food Chem. 2012, 130, 417-424. [CrossRef]

24. Han, R.-P.; Zhang, J.-J.; Han, P.; Wang, Y.-F.; Zhao, Z.-H.; Tang, M.-S. Study of equilibrium, kinetic and thermodynamic parameters about methylene blue adsorption onto natural zeolite. Chem. Eng. J. 2009, 145, 496-504. [CrossRef] 